\title{
The Interaction between Carbon Fiber and Pitch Measured by High Temperature Electron Paramagnetic Resonance
}

\author{
Takeshi Ehara, Haruo Kumagai and Yuzo Sanada \\ (Received January 8, 1992) \\ Metals Research Institute, Faculty of Engineering, \\ Hokkaido University, Sapporo 060, Japan
}

The surface of carbon fiber is one of key properties of composite material with carbon fiber. Interactions between carbon fiber and pitch over the range of carbonization temperature were investigated by in-situ electron paramagnetic resonance (e.p.r.) method.

From in situ e.p.r. measurements for carbon fiber added pitch, the results are summarized as follows: (1) Peak to peak line width of e.p.r. spectra for S1 (petroleum pitch derived fiber carbonized at $873 \mathrm{~K}$ )/ Pitch systems and P2 (PAN derived fiber carbonized at 1173K)/Pitch systems was characteristic at the ambient temperature lower than $673 \mathrm{~K}$. These characteristics were not influenced by kinds of pitches.

(2) Unexpected evidences were found that (i) no obvious differences of the spin concentration between experimental and calculated values for CF/Pitch systems up to $673 \mathrm{~K}$, (ii) at the ambient temperature higher than $673 \mathrm{~K}$, experimental values of spin concentration were lower than calculated ones. Especially S1/Pitch I (isotropic pitch) and P1 (PAN derived fiber carbonized at 873K)/Pitch A (anisotropic pitch) systems were remarkable.

The spin concentrations depended upon the combination of carbon fiber and pitch.

(3) Clear relationship between the spin concentration of carbon fiber added pitch and the degree of graphitization of the carbon fiber was found.

\section{KEYWORDS: High temperature e.p.r., Carbon fiber, Pitch Co-carbonization}

\section{Introduction}

Since electron paramagnetic resonance, e.p.r., (so called electron spin resonance, e.s.r.) in graphite was discovered by Castle ${ }^{1)}$, many researchers have reported the observations of e.p.r. for coal, graphite, carbon and so on $^{2)-5}$. In situ high temperature e.p.r. technique makes clear that carbonization behavior of synthetic polymer, petroleum and coal tar pitches is dependent upon the chemical composition and molecular weight distribution of the parent substance and is also affected on heating condition, circumstance of reaction system and use of organic or inorganic additives ${ }^{6)-9}$ ).

Recently, in situ high temperature e.p.r. technique was applied to monitor the influence of inert materials in pitch during carbonization ${ }^{10)}$. The spin concentration of the pitch increases with increasing silica gel content at the temperature of $773 \mathrm{~K}$. With respect to physical properties of silica gel/pitch system during carbonization, addition of inert materials influences the reaction circumstances of pitch. Amount of transferable hydrogen in pitch decreases also with increasing silica gel content. Transferable hydrogens could contribute stabilization of thermally induced fragments with radicals in the pitch during carbonization reaction. They go also to species having radicals which are surviving in the original pitch. The facts above are able to explain that the addition of silica gel which increases the viscosity of the system prevents convection within the fluid matrix and consequently restricts hydrogen 
transfer reaction in the carbonization system.

On the other hand, oxygen containing functional groups over the surface of silica gel are also capping transferable hydrogen in the pitch. Therefore, active sites with dangling bonds and functional groups over the surface of silica gel are responsible to hydrogen transfer reaction at the interface between silica gel and pitch. Consequently, effective hydrogens to stabilize the radicals in the pitch would be declined with the addition of silica gel, both physically and chemically. Spin concentration measured by high temperature e.p.r. increases resultantly with the addition of silica gel.

The surface of carbon fiber is one of key properties to make carbon/carbon composite. The surface of carbon fiber has not been characterized well by means of e.p.r. because the carbon fiber does not show e.p.r. signal due to the skin effect caused by its high electric conductivity. However, there is still remaining possibility to get information on the surface of carbon fiber with pitch by means of in situ high temperature e.p.r. technique. The purpose in this paper is to investigate interaction between carbon fiber and pitch and is to characterize the surface of carbon fiber by this methodology.

\section{Experimental}

\subsection{Pitch and carbon fiber}

Two kinds of petroleum derived pitch were used as a carbonization medium: one was anisotropic pitch (Pitch A) and another was isotropic one (Pitch I). The carbon fibers used for present study were petroleum pitch derived carbon fibers (S-CFs) and PAN derived ones (P-CFs). The carbon fibers were treated at various temperatures as shown in Table $\mathbf{1}$ under a nitrogen gas flow.

\subsection{E.p.r. measurement}

The carbon fibers were mixed with pitch in an agate mortar, where the weight ratio of carbon fiber was $5 \%$ in each sample. From 15 to $25 \mathrm{mg}$ exactly weighed samples were placed in a $15 \mathrm{~cm}$ long heat-resisting quartz tube with external and internal diameters of 4 and $1.6 \mathrm{~mm}$, respectively. The variation in spin concentration and peak to peak line width was monitored with a Varian E-109 X-band e.p.r. spectrometer equipped with Micro Device high temperature cavity. In all experiments, heating rate was $8 \mathrm{~K} \mathrm{~min}^{-1}$ under a nitrogen gas flow. The e.p.r. spectra were recorded at a microwave power of $0.2 \mathrm{~mW}$ for 7.5 second. The signal stored in a Hewlett-Packard

Table 1. Characteristics of pitch and carbon fiber employed.

\begin{tabular}{|c|c|c|c|c|c|c|c|c|}
\hline \multirow[b]{2}{*}{ Sample } & \multirow{2}{*}{$\frac{\text { H.T.T. }}{\mathrm{K}}$} & \multicolumn{4}{|c|}{ Elemental analysis (wt\%) } & \multicolumn{2}{|c|}{ ESCA } & \multirow{2}{*}{$\frac{\text { Spin conc. }}{X 10^{18} \text { spins } / g}$} \\
\hline & & $\mathrm{C}$ & $\mathrm{H}$ & $\mathrm{N}$ & $(\mathrm{O}+\mathrm{S})$ diff. & $\mathrm{N}_{1 \mathrm{~S}} / \mathrm{C}_{1 \mathrm{~S}}$ & $\mathrm{O}_{1 \mathrm{~S}} / \mathrm{C}_{1 \mathrm{~S}}$ & \\
\hline Pitch A & ---- & 94.85 & 4.20 & 0 & 0.95 & & & 14.2 \\
\hline Pitch I & $\cdots$ & 91.86 & 5.31 & 0 & 2.83 & & & 2.21 \\
\hline \multicolumn{9}{|l|}{ S-CFs } \\
\hline S1 & 873 & 92.56 & 3.09 & 0 & 4.35 & & & 94.2 \\
\hline S2 & 1273 & 98.01 & 0.25 & 0 & 1.74 & & & --- \\
\hline S3 & 1773 & 98.94 & 0 & 0 & 1.06 & & & ---- \\
\hline S4 & 2273 & 99.80 & 0 & 0 & 0.20 & & & $---\cdot$ \\
\hline S5 & 3193 & 99.85 & 0 & 0 & 0.15 & & & $-\cdots$ \\
\hline \multicolumn{9}{|l|}{ P-CFs } \\
\hline P1 & 873 & 66.90 & 2.21 & 19.27 & 11.62 & 0.30 & 0.39 & 33.1 \\
\hline $\mathrm{P} 2$ & 1173 & $-\cdots$ & ---- & $-\cdots--$ & ---- & 0.13 & 0.33 & Dysonian \\
\hline P3 & 1773 & $-\cdots$ & $\cdots$ & $-\cdots-$ & $-\cdots--$ & 0 & 0.16 & ---- \\
\hline P4 & 2273 & $\ldots$ & ---- & -..-- & ----- & 0 & 0.07 & ---- \\
\hline
\end{tabular}


computer for further integration to get absorption area. The absolute values of spin concentration, Ns, [spins/g] were calculated from e.p.r. absorption area. It was expressed by following equation:

$$
N_{S}=N_{S 0} \times \frac{A_{S}}{A_{S 0}} \times \frac{1}{W} \times \frac{T}{T_{0}}
$$

where Nso [spins/g] is the numbers of spin in standard sample. As and Aso represent the absorption area for sample and standard, respectively. $\mathrm{W}[\mathrm{g}]$ is the weight of sample. $\left(\mathrm{T} / \mathrm{T}_{0}\right)$ means the correction for the Boltzmann factor. The linear weight loss was assumed over the range of observation temperature in the same way as described in the previous paper ${ }^{11), 12)}$.

Measurements of spin concentration for original samples at room temperature were performed with the separate samples evacuated to a pressure of $2 \times 10^{-5}$ Torr.

\section{Results and Discussion}

\subsection{Characteristics and properties of sample}

Table 1 shows the characteristics of the samples. Pitch A (optically anisotropic) and Pitch I (optically isotropic) had almost similar values for elemental analysis. For petroleum pitch based carbon fibers, S-CFs, a trend is recognized that carbon content increased and hydrogen content decreased as the increase of heat-treatment temperature. Elemental analysis for PAN based carbon fibers, P-CFs treated over $1173 \mathrm{~K}$ were not available because they do not burn in elemental analyzer. The comparison of the elemental composition for P-CFs was carried out by means of ESCA. The ratio of nitrogen $\left(\mathrm{N}_{1 \mathrm{~s}} / \mathrm{C}_{1 \mathrm{~s}}\right)$ and oxygen $\left(\mathrm{O}_{1 \mathrm{~s}} / \mathrm{C}_{1 \mathrm{~s}}\right)$ decreased as increase of heat-treatment temperature as shown in Table 1.

\subsection{Spin concentration of samples at room temperature}

Spin concentration, $\mathrm{N}_{\mathrm{S}}$, for the original samples at room temperature is also shown in Table 1. Pitch A had value of $\mathrm{N}_{\mathrm{S}}$ about 7 times larger than that of Pitch $\mathrm{I}$. For the carbon fibers, S1 and P1 treated at lowest temperature so far tested showed symmetrical e.p.r. absorption in shape and enabled to calculate the value of spin concentration. P2 showed Dysonian type e.p.r. absorption in shape, and spin concentration was not able to be calculated. For all other carbon fibers treated at high temperature levels, e.p.r. absorption could not be observed due to skin effect.

\subsection{Temperature dependence of peak to peak line width}

Figure 1 shows temperature dependence of peak to peak line width of e.p.r. signals, $\triangle H_{P P}$, for S-CF/Pitch A systems. The system with S4/Pitch A showed almost the same change in $\triangle H_{p p}$ as Pitch $A$ alone, while S4 alone did not have e.p.r. absorption at room temperature. The system with $\mathrm{S} 1$ gave the values between those of Pitch $\mathrm{A}$ alone and $\mathrm{S} 1$ alone up to 673K. S-CF/Pitch I systems showed a similar tendency as $\mathrm{S}-\mathrm{CF} / \mathrm{Pitch} \mathrm{A}$ systems.

The variation of $\triangle H_{P P}$ for P-CF/Pitch A systems is illustrated in Figure 2. The system with P1 showed

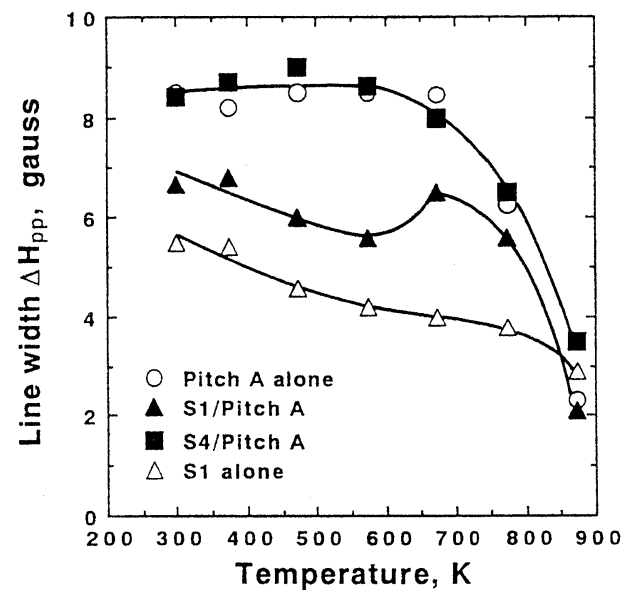

Fig. 1 Temperature dependence of peak to peak line width of S-CFs/Pitch A systems

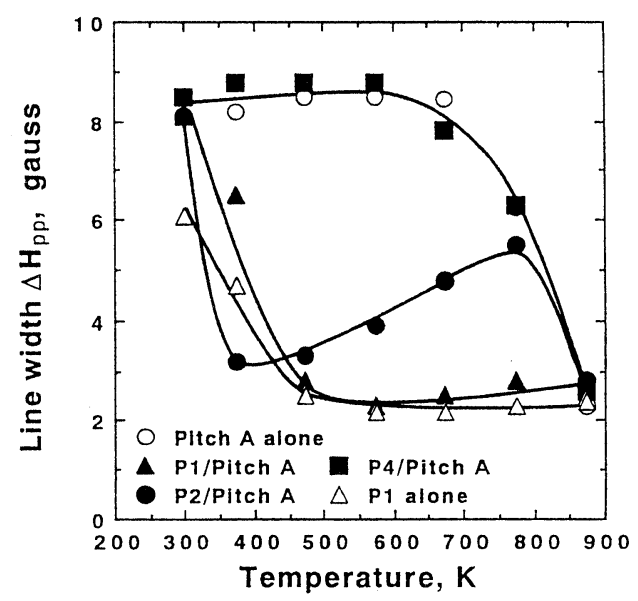

Fig. 2 Temperature dependence of peak to peak line width of P-CFs/Pitch A systems. 
almost similar change to that of P1 alone. On the other hand, P4/Pitch A system showed almost the same change as Pitch $\mathrm{A}$ alone. The system with $\mathrm{P} 2$ having Dysonian type e.p.r. absorption at room temperature was different from the other systems over the temperature range between $473 \mathrm{~K}$ and $673 \mathrm{~K}$. In the case of P-CF/Pitch I systems, similar tendency was also observed with P-CF/Pitch A systems.

The temperature dependence of $\triangle H_{P P}$ for the pitches added with carbon fibers derived from petroleum pitch and PAN is not similar as seen in Figure 1 and Figure 2. Therefore, the difference of cocarbonization behaviors between S-CF and P-CF might be provided information of the pitch precursors by in situ e.p.r. method.

Line width, $\triangle H_{P P}$, is influenced not only by interaction between the electron spin and external magnetic field, but also by interaction of electron spin with the environment inside the sample. From the line width of e.p.r. spectra of the system, information about the spin environment is obtained. E.p.r. lines can be broadened homogeneously when all spins have the same environment and broadened inhomogenously when spins are in different environments. The reduction in the symmetry of the radical leads to an increase in the spin lattice relaxation time, and consequently to narrowing of the line width.

It is not easy to discuss the detail on the carbonization system because the sample systems described above are too complex. It could say safely only that the addition of carbon fiber. affects environments of spins in the pitch. This facts lead to characterization of carbon fiber surface through the changes of radicals in the pitch.

\subsection{Temperature dependence of spin concentration}

\subsubsection{Low temperature heat-treated CF/Pitch systems}

The effects of interaction between carbon fiber and pitch on the change in spin concentration were examined for the samples of $\mathrm{S} 1$ and P1. Figure 3 shows the temperature dependences of spin concentration obtained from respective S1/Pitch $\mathrm{A}$ and I systems together with those of calculated values. Calculated value $\mathrm{N}_{S}$ (calc.) [spins/g-sample] is derived as follows:

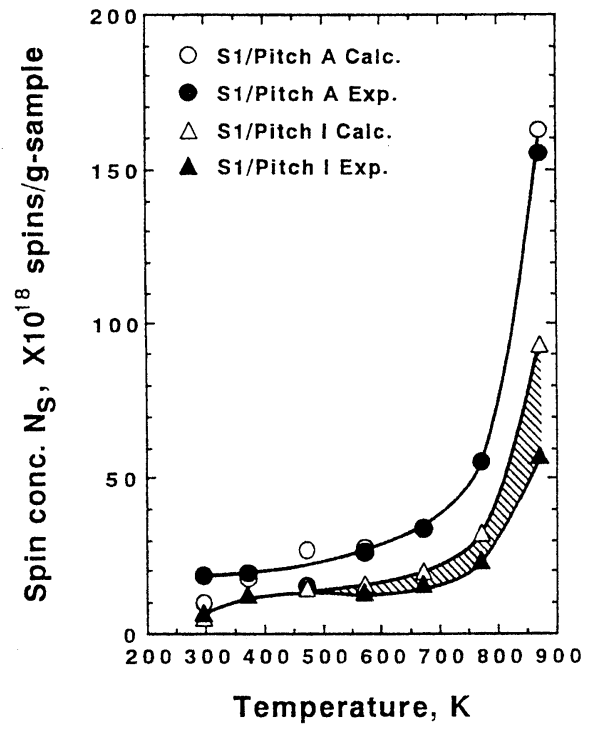

Fig. 3 Comparison between the experimental values and calculated values of spin concentration for S1/Pitch A and I systems

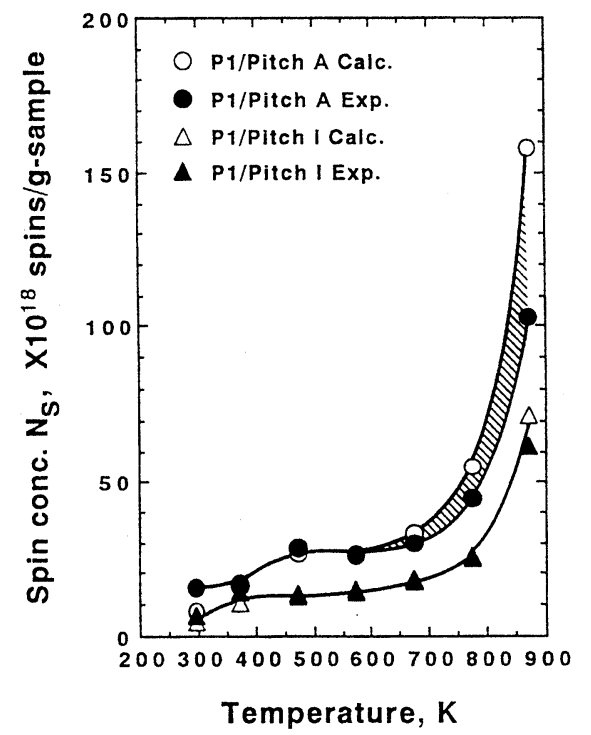

Fig. 4 Comparison between the experimental values and calculated values of spin concentration for P1/Pitch $\mathrm{A}$ and I systems

$$
\mathrm{N}_{S}(\text { calc. })=0.05 \times \mathrm{N}_{S}(\mathrm{CF})+0.95 \times \mathrm{N}_{\mathrm{S}}(\text { Pitch })
$$

where $\mathrm{N}_{\mathrm{S}}(\mathrm{CF})$ and $\mathrm{N}_{\mathrm{S}}(\mathrm{Pitch})$ [spins/g] represent the spin concentration of carbon fiber and pitch alone, respectively. Figure 4 shows comparison between the 
experimental and calculated values for P1 systems. Let us assume that the extent of interactions between carbon fibers and pitches are given as the difference between experimental and calculated spin concentrations. The interactions were not remarkable up to $723 \mathrm{~K}$ for both $\mathrm{S} 1$ and $\mathrm{P} 1$. Contrary, obvious interactions were observed at $873 \mathrm{~K}$ for both $\mathrm{S} 1$ and $\mathrm{P} 1$. The interactions depend on combinations of carbon fibers and pitches. S1 or P1 prevents noticeable increase of spin concentration of Pitch $\mathrm{I}$ or Pitch $\mathrm{A}$, respectively.

It has been shown that the change in spin concentration with reaction time (or temperature) in A240 pitch alone and the pitch/silica systems. The spin concentration in pitch even at a weight ratio of 100:1 was remarkably increased ${ }^{10)}$. As is shown in Figure 3 and Figure 4, no obvious differences of the spin concentration between experimental and calculated values in the pitch up to $673 \mathrm{~K}$ for all cases. It is noted that the spin concentration values, $\mathrm{N}_{\mathrm{S}}$ (calc), for $\mathrm{S} 1 /$ pitch I and P1/pitch A systems become larger than those of experimental ones, nevertheless the expected values by calculation are low.

\subsubsection{Heat-treatment temperature of carbon fiber}

No favorable information is obtained from $\triangle H_{P P}$ for the carbon fibers treated at higher temperatures as shown in Figure 1 and Figure 2. Thus, surface characteristics of the carbon fibers heat-treated at various temperature have also been attempted to observe through the change in spin concentration of pitches added with carbon fibers.

Assuming that the spin concentration of carbon fiber is almost constant during heating, the spin concentration of pitch, $\mathrm{N}_{\mathrm{s}}$ '(calc.) [spins/g-pitch] are obtained by:

$$
\mathrm{N}_{\mathrm{S}^{\prime}}(\text { calc. })=\left[\mathrm{N}_{\mathrm{S}}(\exp .)-0.05 \times \mathrm{N}_{\mathrm{S}}(\mathrm{CF})\right] / 0.95
$$

where $\mathrm{N}_{\mathrm{s}}$ (exp.) represents the spin concentration of experimental value for carbon fiber and pitch system. $\mathrm{N}_{\mathrm{S}}(\mathrm{CF})$ represents the spin concentration of carbon fiber alone. The assumption stands upon the facts that (1) $\mathrm{CF}$ is stable because it has been already heat-treated over the temperature range of $873-3193 \mathrm{~K}$ for $\mathrm{S} 1$ and 873 - $2273 \mathrm{~K}$ for P1, respectively; (2) pitch behaves pyrolyse with chemical reactions such as bond scissions and recombination so on, and alters its spin concentration.

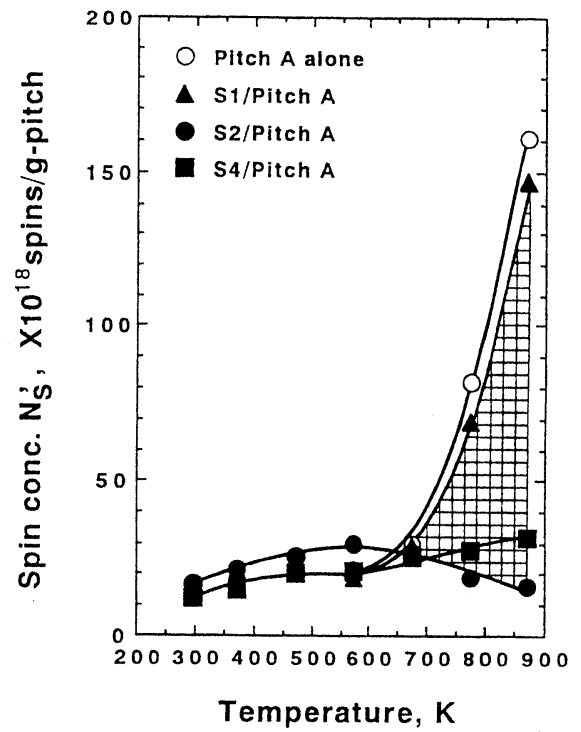

Fig. 5 Temperature dependence of spin concentration for S-CFs/Pitch A systems

The effects of S-CFs addition on the spin concentration of Pitch $\mathrm{A}$ are shown in Figure 5. Above $723 \mathrm{~K}$, the changes of spin concentration of pitch $\mathrm{A}$ become noticeable with addition of S-CFs. Especially the systems with S-CFs treated at $1273 \mathrm{~K}$ and $2273 \mathrm{~K}$ are obvious.

The spin concentrations at $873 \mathrm{~K}$ of ambient temperature for S-CFs/Pitch I systems had a similar variation to those of $\mathrm{S}-\mathrm{CFs} / \mathrm{Pitch} \mathrm{A}$, that is;

$($ Pitch I alone $)>($ S1/Pitch I $)>($ S4/Pitch I $)>($ S2/Pitch I $)$

The temperature dependence of spin concentration of Pitch A with the addition of P-CFs is shown in Figure 6. P-CFs prevent also the increase of Pitch A's spin concentration above $723 \mathrm{~K}$. Their influences are very remarkable at $873 \mathrm{~K}$ as same as $\mathrm{S}-\mathrm{CFs} / \mathrm{Pitch} \mathrm{A}$ system. Comparing the systems for P-CFs and S-CFs in pitch $A$, the values of spin concentration for S2/Pitch A systems declined at $873 \mathrm{~K}$. Although no remarkable declinings are observed for the systems of P-CFs above $723 \mathrm{~K}$.

For P-CFs/Pitch I systems, there were the same trend to the systems mentioned above. The influence of the carbon fibers in the pitch became appeared from the temperature at $723 \mathrm{~K}$ and became most remarkable at $873 \mathrm{k}$. The order of spin concentration at measurement 


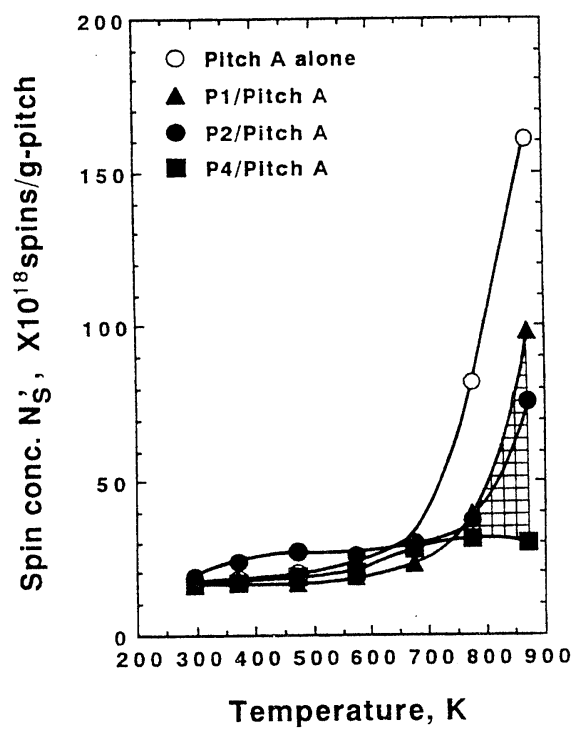

Fig. 6 Temperature dependence of spin concentration for P-CFs/Pitch A systems

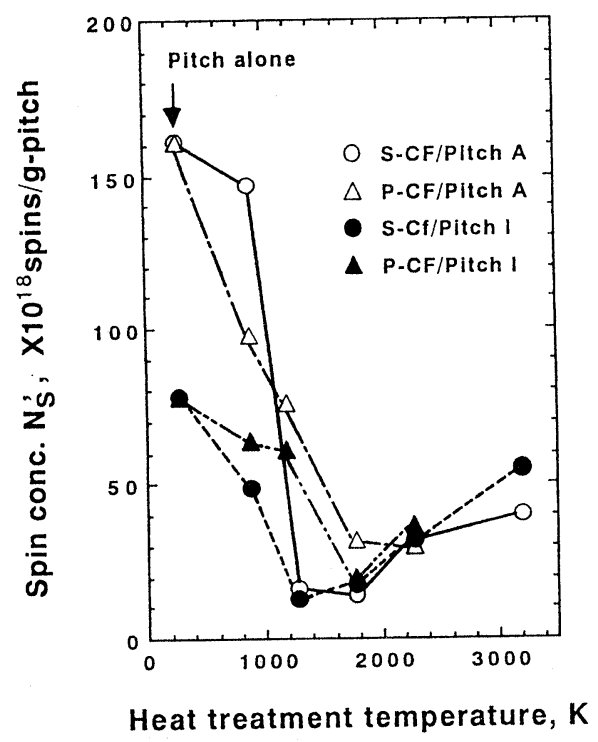

Fig. 7 H.T.T. of CFs vs. spin concentration of CFs/ Pitch systems at $873 \mathrm{~K}$

temperature $873 \mathrm{~K}$ was that:

$($ Pitch I alone $)>($ P1/Pitch $\mathrm{I}) \simeq(\mathrm{P} 2 /$ Pitch $\mathrm{I})>(\mathrm{P} 4 / \mathrm{Pitch} \mathrm{I})$

It is certain that the active sites exist more or less over the surface of carbon fiber. From the experiments on the influence of inert materials in pitch at the vicinity of $673 \mathrm{~K}$, spin concentration of the pitch increases with addition of inert materials such as silica gel, carbon black powders and so on ${ }^{10)}$. During pyrolysis reaction, transferable hydrogens, which are able to stabilize radicals in the pitch, are capped by the special sites located at the surface of the inert materials. Resultantly, the increase of spin concentration of the inert material added pitch is larger than that of the case of single carbonization of pitch. Oxygen containing functional groups over the surface of inert materials are also responsible for the increase of spin concentration in pitch as described above. Question has come why the spin concentration of pitch decreases with the addition of carbon fiber as referred to the case of silica gel and carbon black. This is not clear at the moment. Electrical conductivity of the sample in e.p.r. tube caused by the large aspect ratio of the ground carbon fiber in pitch may result to one of reasons. It is also thought that special alignment of lamella in pitch may occur over the surface of carbon fiber. Further experiments will make clear the problems.

The changes of the spin concentration measured at $873 \mathrm{~K}$ for all cases of pitch-carbon fiber system versus heat-treatment temperature of carbon fibers are shown in Figure 7. In every system, as increasing heattreatment temperature of carbon fiber, the values of spin concentration of the pitches decrease remarkably at first, reaching a minimum, then they had a little increase. These results suggest that there are clear relationships between the spin concentration of carbon fiber added pitch and the degree of graphitization of the carbon fiber.

\section{Summary}

From in situ e.p.r. measurements for carbon fiber added pitch, the results are summarized as follows: (1) Peak to peak line width of e.p.r. spectra for S1 (petroleum pitch derived fiber carbonized at 873K)/Pitch systems and P2 (PAN derived fiber carbonized at $1173 \mathrm{~K}$ )/Pitch systems was characteristic at the ambient temperature lower than $673 \mathrm{~K}$. These characteristics were not influenced by kinds of pitches. (2) Unexpected evidences were found that (i) no obvious differences of the spin concentration between experimental and calculated values for $\mathrm{CF} / \mathrm{Pitch}$ 
systems up to $673 \mathrm{~K}$, (ii) at the ambient temperature higher than $673 \mathrm{~K}$, experimental values of spin concentration were lower than calculated ones. Especially S1/Pitch I (isotropic pitch) and P1 (PAN derived fiber carbonized at $873 \mathrm{~K}$ )/Pitch A (anisotropic pitch) systems were remarkable.

The spin concentrations depended upon the combination of carbon fiber and pitch.

(3) Clear relationship between the spin concentration of carbon fiber added pitch and the degree of graphitization of the carbon fiber was found.

\section{References}

1) J.G. Castle, Jr., Phys. Rev. 92 (1953) 1063.

2) J.G. Castle, Jr., Phys. Rev. 94 (1954) 1410.

3) D.J.E. Ingram and J.E. Bennett, Phil. Mag. 45 (1954) 545.

4) L.S. Singer, Proc. 5th Conf. Carbon, Vol. 2 (1963)

\section{Pergamon.}

5) L.S. Singer and G. Wagoner, Proc. 5th Conf. Carbon, Vol. 2 (1963) 65, Pergamon.

6) S. Toyoda, Report of Resources Research Institute, "Magnetochemical studies of coals and their related substances (part 3)" (1969).

7) T. Yokono, S. Iyama, Y. Sanada and K. Makino, Fuel 64 (1985) 1014.

8) T. Yokono, S. Iyama, Y. Sanada, S. Simokawa and E. Yamada, Fuel 65 (1986) 1701.

9) T. Yokono, S. Iyama, Y. Sanada and K. Makino, Carbon 22 (1984) 624.

10) T. Kaneko, L.E. Henao, T. Yokono, T. Ehara and Y. Sanada, J. Matl. Sci. Letters 9 (1990) 351.

11) T. Obara, T. Yokono, Y. Sanada and H. Marsh, Fuel 64 (1985) 995.

12) T. Yokono, T. Kohno and Y. Sanada, Fuel 64 (1985) 411. 